\title{
Impairment of zebrafish reproduction upon exposure to TDCPP
}

\author{
Xiecheng Liu, Guofeng Jia* \\ Department of Health Management, Qingdao Municipal Hospital, Qingdao 266021, \\ Shandong, China.
}

*Corresponding author: Dr. Guofeng Jia

\begin{abstract}
TDCPP is one of the most common organophosphate flame retardant, which has been widely used in many products. It has been detected in the environment and biota; however, its potential affect to the wildlife and human health remains largely unknown. In this study, we aimed to investigate the effect of long-term exposure to TDCPP on fish reproduction. Zebrafish eggs were treated with various concentration of TDCPP $(0,1,10$ and $100 \mu \mathrm{g} / \mathrm{L})$ from 1 day post-fertilization (hpf) to 6 months. The fecundity of female fish was significantly decreased as indicated by reduced embryos production. The egg quality was decreased and the malformation rates were increased in the F1 generation. Taken together, long-term exposure to TDCPP affects the reproduction of zebrafish.
\end{abstract}

Keywords: TDCPP; Reproduction; Zebrafish 


\section{Background}

Tris(1,3-dichloro-2-propyl) phosphate (TDCPP) is an orgnophosphate flame retardant, which has been widely applied in the products of resins, latexes and polyurethane foams in the furniture [1-5]. TDCPP is easily to be released into the environment because its non-chemically bounding property to the household items $[3,6-10]$. Thus, TDCPP can cause pervasive contamination in the air, surface and ground water [11-15]. TDCPP is relatively stable in water because it is lipophilic and therefore hard to be decomposed in the environment and could lead to bioaccumulate in the aquatic animals [4, 16-18].

Previous studies have reported that TDCPP could affect the sex hormone levels in human cells and induce the transcriptional expression of estrogenic receptors and related genes in zebrafish [19]. TDCPP affects the estrogenic activity and interrupts endocrine levels in fish [20-22], however, the effect of TDCPP on the reproductively in TDCPP exposure on zebrafish reproduction, especially in a long-term exposure period, which most reflects the affection of TDCPP pollution in the aquatic environment.

\section{Materials and methods}

\section{Chemicals}


TDCPP, [(1,3-dichloro-2-propyl) phosphate] (analytical standard) was purchased from Sigma Aldrich. Stock solutions were prepared in dimethyl sulfoxide (DMSO, purity $>99 \%$ ) and stored at $-20^{\circ} \mathrm{C}$.

\section{Zebrafish maintenance and TDCPP treatment}

Wild type zebrafish were raised as described previously [23-27]. The fish were kept at $28 \pm 0.5^{\circ} \mathrm{C}$ in a $14 \mathrm{~h}$ light/10 h dark cycle. Fish were naturally crossed and eggs were collected. Five hundred fertilized zebrafish embryos were treated with TDCPP $(0,1,10$ and $100 \mu \mathrm{g} / \mathrm{L})$ in 1 -L tanks. The zebrafish larvae were put into 2.8 -L aquariums at $20 \mathrm{dpf}$. Each exposure groups and controls had three replicates and received $0.001 \%(\mathrm{v} / \mathrm{v})$ DMSO. Fish were mated in groups weekly to evaluate the reproductive capacity. Fecundity was calculated as the cumulative average number of embryos produced per female each time.

\section{Measurement of hormone concentrations}

The estradiol and testosterone levels were examined in the blood samples of TDCPP-treated zebrafish as previously described [28-30]. In brief, fish were euthanized in MS-222 (200 mg/L), the blood samples from caudal vein were collected, centrifuged at $5000 \times \mathrm{g}$ for $10 \mathrm{~min}$ at $4{ }^{\circ} \mathrm{C}$. The supernatant was collected for hormones extraction. 


\section{Data analysis}

All analyses were carried out using SPSS 18.00 (SPSS, Chicago, IL, USA). Differences between the control and TDCPP-treated group were assessed by one-way analysis of ANOVA followed by a Tukey's test. All data were presented as the mean $\pm \mathrm{SE}, \mathrm{P}<$ 0.05 was considered statistically significant.

\section{Results}

\section{Toxicological effects of TDCPP exposure to zebrafish}

The hatching, malformation and survival rates of the F1 generation were recorded from $1 \mathrm{dpf}$ to $5 \mathrm{dpf}$. There was no significantly difference in the F1 eggs derived from the P0 fish exposed to 1 and $10 \mu \mathrm{g} / \mathrm{L}$ TDCPP. However, the malformation rate in the F1 eggs derived from P0 fish that were treated with $100 \mu \mathrm{g} / \mathrm{L}$ TDCPP was significantly increased (Data not shown).

The survival rates in the TDCPP-exposed P0 fish had no significant difference during the six months of treatment. As shown in Fig. 1, the average numbers of embryos produced were significantly decreased in the groups treated with 10 and $100 \mu \mathrm{g} / \mathrm{L}$ TDCPP compared to the controls.

\section{Hormones levels in TDCPP exposed fish}


Compared to the controls, the estradiol (E2) and testosterone (T) levels in female

fish treated with 10 and $100 \mu \mathrm{g} / \mathrm{L}$ TDCPP were increased (Fig. 2); however, the E2 and T levels in the male fish treated with TDCPP had no significant changes (Fig. 3).

\section{Discussion}

In this study, we investigated the effects of long-term exposure to TDCPP on plasma hormone levels in zebrafish, which is consistent with the previous studies that report TDCPP alters steroidogenesis in fish [31-35]. In addition, we observed that exposed to high concentration of TDCPP reduced the embryos production and caused higher malformation rates in the F1 eggs. There results confirmed that longterm exposure to environmental concentrations of TDCPP affect the fish reproductive system [35-40].

We also found that the E2 and T levels were higher in the female but not in the male fish exposed to TDCPP, which indicated that TDCPP might have gender-specific effects on the zebrafish steroidgenesis. These data were in consistent with the previous studies that endocrine disrupting chemicals have estrogenic activity and could affect the sex hormones in fish in a gender-specific manner [41-48]. The toxicity of TDCPP treatment was investigated in P0 and F1 zebrafish. We observed the malformation rate in F1 embryos that derived from P0 fish treated with TDCPP was increased. This data suggested that TDCPP had developmental toxicity to the offspring when the parental fish were treated with TDCPP. Thus, our 
results suggested that long-term exposure to environmental concentration of TDCPP to P0 fish resulted in developmental toxicity to the F1 embryos.

Taken together, our study confirmed that chronic treatment with environmental concentrations of TDCPP impaired zebrafish reproductive system [5, 32, 35, 38, 49, 50]. However, the effects of TDCPP on the sex hormone levels in fish still need further investigation. 


\section{Figure Legends}

Figure 1. The productions of embryos in zebrafish treated with TDCPP in

different concentration. ${ }^{*} \mathrm{p}<0.05$ present significance between exposed group and the controls.

Fig. 1

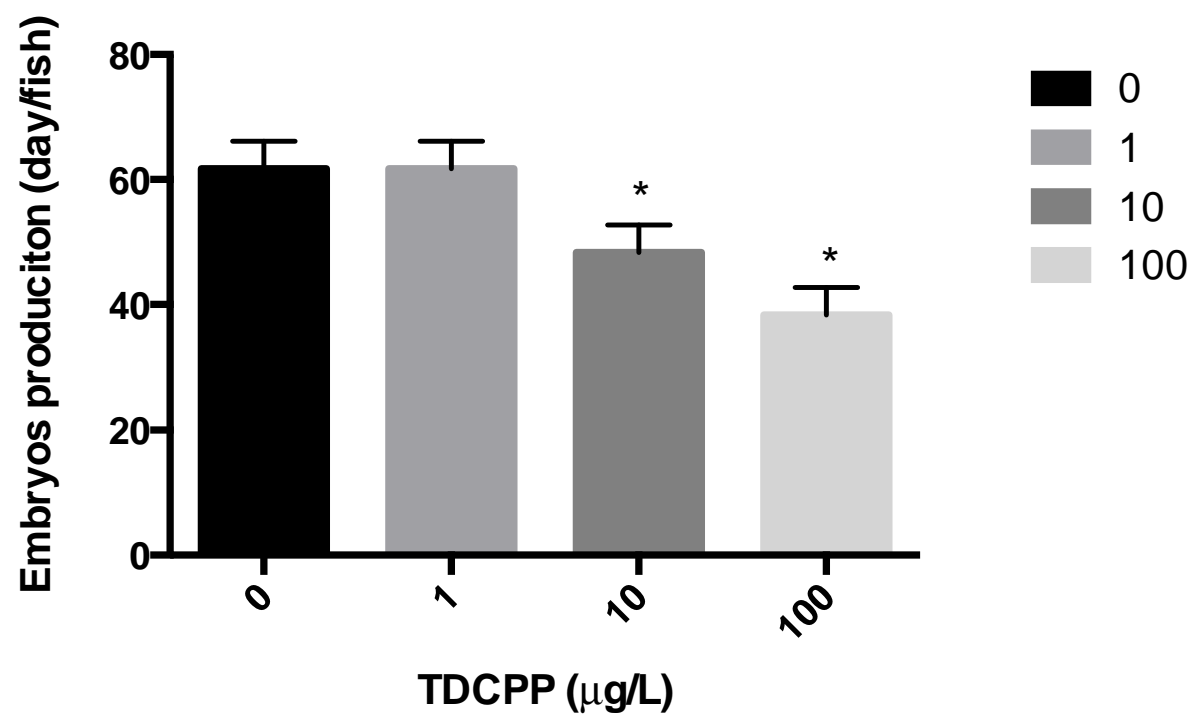

Figure 2. The estradiol (E2) and testosterone (T) contents in the female fish treated with TDCPP. ${ }^{*} \mathrm{p}<0.05$ present significance between exposed group and the controls. 
Fig. 2

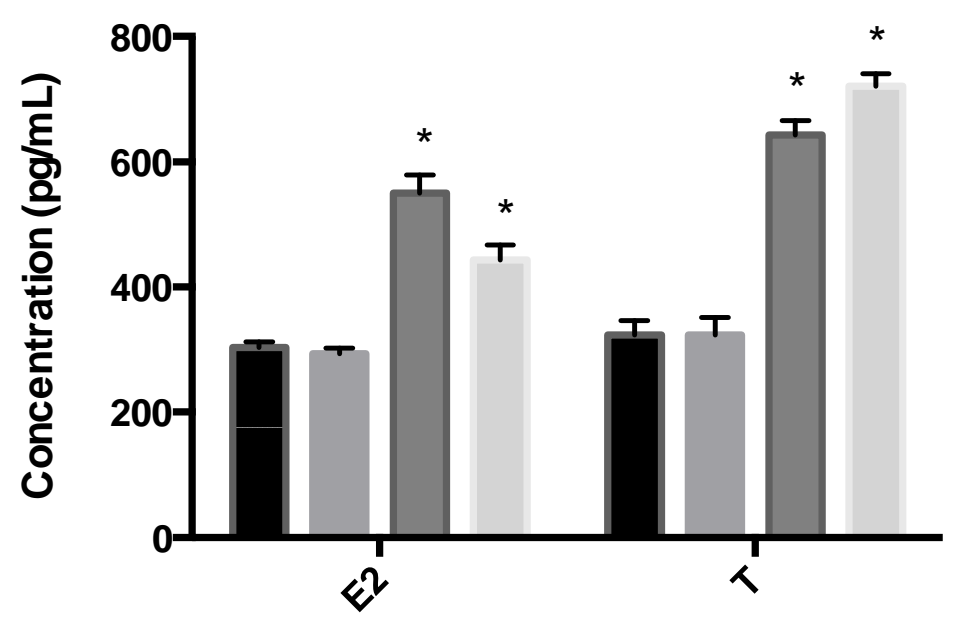

$0 \mu \mathrm{g} / \mathrm{L}$

$1 \mu \mathrm{g} / \mathrm{L}$

$\square 10 \mu \mathrm{g} / \mathrm{L}$

$100 \mu \mathrm{g} / \mathrm{L}$

Figure 3. The estradiol (E2) and testosterone (T) contents in the male fish

treated with TDCPP.

Fig. 3

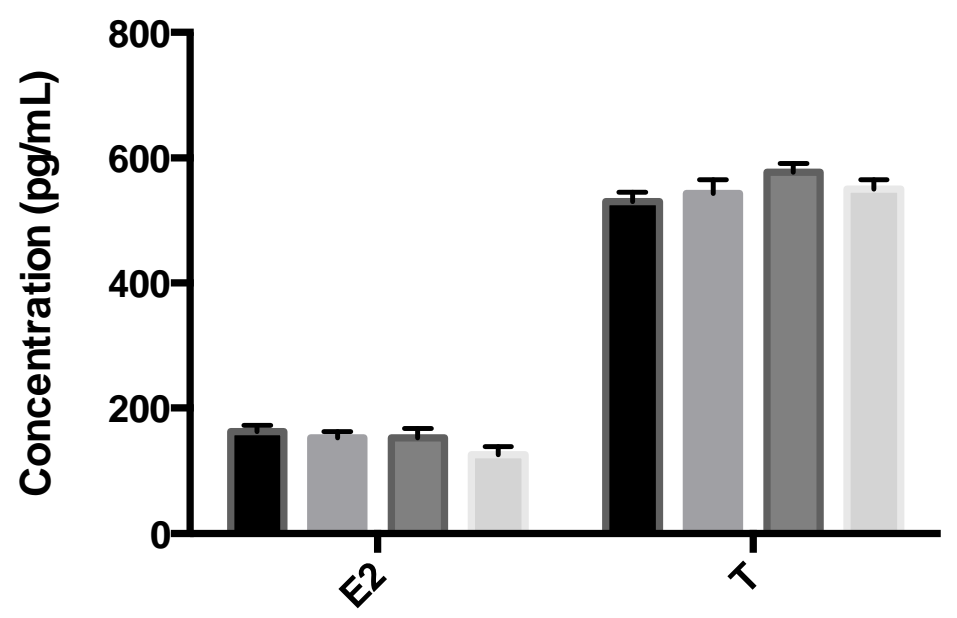

$0 \mu \mathrm{g} / \mathrm{L}$

$1 \mu \mathrm{g} / \mathrm{L}$

$\square 10 \mu \mathrm{g} / \mathrm{L}$

$100 \mu \mathrm{g} / \mathrm{L}$ 


\section{References}

1. Mao, R., et al., AIBP Limits Angiogenesis Thorough $\gamma$-Secretase-Mediated Upregulation of Notch Signaling. Circulation Research, 2017: p. CIRCRESAHA.

\subsection{4 .}

2. Meeker, J.D., et al., Urinary metabolites of organophosphate flame retardants: temporal variability and correlations with house dust concentrations. Environmental health perspectives, 2013.121(5): p. 580.

3. Dodson, R.E., et al., After the PBDE phase-out: a broad suite of flame retardants in repeat house dust samples from California. Environmental science \& technology, 2012. 46(24): p. 13056-13066.

4. Van der Veen, I. and J. de Boer, Phosphorus flame retardants: properties, production, environmental occurrence, toxicity and analysis. Chemosphere, 2012. 88(10): p. 1119-1153.

5. Dishaw, L.V., et al., Developmental exposure to organophosphate flame retardants elicits overt toxicity and alters behavior in early life stage zebrafish (Danio rerio). Toxicological Sciences, 2014. 142(2): p. 445-454.

6. $\mathrm{Gu}, \mathrm{Q}$., et al., Genetic ablation of solute carrier family 7a3a leads to hepatic steatosis in zebrafish during fasting. Hepatology, 2014. 60(6): p. 1929-1941.

7. Kawagoshi, Y., S. Nakamura, and I. Fukunaga, Degradation of organophosphoric esters in leachate from a sea-based solid waste disposal site. Chemosphere, 2002. 48(2): p. 219-225. 
8. Kucharska, A., et al., Non-invasive biomonitoring for PFRs and PBDEs: new insights in analysis of human hair externally exposed to selected flame retardants. Science of The Total Environment, 2015. 505: p. 1062-1071.

9. Marklund, A., B. Andersson, and P. Haglund, Screening of organophosphorus compounds and their distribution in various indoor environments.

Chemosphere, 2003. 53(9): p. 1137-1146.

10. Bacaloni, A., et al., Liquid chromatography/tandem mass spectrometry determination of organophosphorus flame retardants and plasticizers in drinking and surface waters. Rapid communications in mass spectrometry, 2007. 21(7): p. 1123-1130.

11. Sparling, D.W., Ecotoxicology Essentials: Environmental Contaminants and Their Biological Effects on Animals and Plants. 2016: Academic Press.

12. Cristale, J., et al., Assessment of flame retardants in river water using a ceramic dosimeter passive sampler. Environmental pollution, 2013. 172: p. 163-169.

13. Demeneix, B., Toxic Cocktail: How Chemical Pollution Is Poisoning Our Brains. 2017: Oxford University Press.

14. Cordner, A., Toxic Safety: Flame Retardants, Chemical Controversies, and Environmental Health. 2016: Columbia University Press.

15. Venier, M., A. Salamova, and R.A. Hites, Halogenated flame retardants in the Great Lakes environment. Accounts of chemical research, 2015. 48(7): p. $1853-1861$

16. Richardson, S.D. and S.Y. Kimura, Water analysis: emerging contaminants and current issues. Analytical chemistry, 2015. 88(1): p. 546-582. 
17. Luna, R.F., Non-targeted chemical analysis for the identification of persistent organic compounds in treated wastewater. 2016, San Diego State University.

18. Iqbal, M., et al., Legacy and emerging flame retardants (FRs) in the freshwater ecosystem: A review. Environmental research, 2017. 152: p. 26-42.

19. Zhai, G., et al., Sept6 is required for ciliogenesis in Kupffer's vesicle, the pronephros, and the neural tube during early embryonic development. Molecular and cellular biology, 2014. 34(7): p. 1310-1321.

20. Song, G., et al., Effective gene trapping mediated by Sleeping Beauty transposon. PloS one, 2012. 7(8): p. e44123.

21. Dirtu, A.C., et al., Analytical methods for selected emerging contaminants in human matrices - a review. Analytical and bioanalytical chemistry, 2012. 404(9): p. 2555-2581.

22. OR, P., TSCA Work Plan Chemical Problem Formulation and Initial Assessment Chlorinated Phosphate Ester Cluster Flame Retardants. 2015.

23. Yang, X., et al., Nucleoporin 62-like protein activates canonical Wnt signaling through facilitating the nuclear import of $\beta$-catenin in zebrafish. Molecular and cellular biology, 2015. 35(7): p. 1110-1124.

24. Gu, Q., et al., Generation and characterization of a transgenic zebrafish expressing the reverse tetracycline transactivator. Journal of genetics and genomics, 2013. 40(10): p. 523-531.

25. Haffter, P., et al., The identification of genes with unique and essential functions in the development of the zebrafish, Danio rerio. Development, 1996. 123(1): p. 1-36. 
26. Kawakami, K., A. Shima, and N. Kawakami, Identification of a functional transposase of the Tol2 element, an Ac-like element from the Japanese medaka fish, and its transposition in the zebrafish germ lineage. Proceedings of the National Academy of Sciences, 2000. 97(21): p. 11403-11408.

27. Darland, T. and J.E. Dowling, Behavioral screening for cocaine sensitivity in mutagenized zebrafish. Proceedings of the National Academy of Sciences, 2001. 98(20): p. 11691-11696.

28. Judd, H.L., et al., Endocrine function of the postmenopausal ovary: concentration of androgens and estrogens in ovarian and peripheral vein blood. The Journal of Clinical Endocrinology \& Metabolism, 1974.39(6): p. 1020-1024.

29. Ferrini, R.L. and E. Barrett-Connor, Sex hormones and age: a cross-sectional study of testosterone and estradiol and their bioavailable fractions in community-dwelling men. American journal of epidemiology, 1998. 147(8): p. $750-754$.

30. Harman, S.M., et al., Longitudinal effects of aging on serum total and free testosterone levels in healthy men. The Journal of Clinical Endocrinology \& Metabolism, 2001. 86(2): p. 724-731.

31. Kwon, B., et al., Effects of tris (2-butoxyethyl) phosphate exposure on endocrine systems and reproduction of zebrafish (Danio rerio). Environmental Pollution, 2016. 214: p. 568-574.

32. Dishaw, L.V., et al., Exposures, mechanisms, and impacts of endocrine-active flame retardants. Current opinion in pharmacology, 2014. 19: p. 125-133. 
33. Greaves, A.K. and R.J. Letcher, Comparative body compartment composition and in ovo transfer of organophosphate flame retardants in North American Great Lakes herring gulls. Environmental science \& technology, 2014. 48(14): p. $7942-7950$.

34. Meeker, J.D., et al., Exploratory analysis of urinary metabolites of phosphoruscontaining flame retardants in relation to markers of male reproductive health. Endocrine disruptors, 2013. 1(1): p. e26306.

35. Naderi, M., M.Y. Wong, and F. Gholami, Developmental exposure of zebrafish (Danio rerio) to bisphenol-S impairs subsequent reproduction potential and hormonal balance in adults. Aquatic toxicology, 2014. 148: p. 195-203.

36. Zhong, L., et al., Investigation of effect of $17 \alpha$-ethinylestradiol on vigilin expression using an isolated recombinant antibody. Aquatic toxicology, 2014. 156: p. 1-9.

37. Nash, J.P., et al., Long-term exposure to environmental concentrations of the pharmaceutical ethynylestradiol causes reproductive failure in fish.

Environmental health perspectives, 2004.112(17): p. 1725.

38. Babrauskas, V., et al., Flame retardants in furniture foam: benefits and risks. Fire Safety Science, 2011. 10: p. 265-278.

39. Farhat, A., et al., In ovo effects of two organophosphate flame retardantsTCPP and TDCPP_on pipping success, development, $m R N A$ expression, and thyroid hormone levels in chicken embryos. toxicological sciences, 2013. 134(1): p. 92-102. 
40. Organization, W.H., Flame retardants: tris (chloropropyl) phosphate and tris (2-chloroethyl) phosphate. 1998.

41. Meng, S., et al., Transdifferentiation Requires iNOS ActivationNovelty and Significance. Circulation Research, 2016. 119(9): p. e129-e138.

42. Newbold, R.R., et al., Effects of endocrine disruptors on obesity. International journal of andrology, 2008. 31(2): p. 201-208.

43. Filby, A.L., et al., Gene expression profiles revealing the mechanisms of antiandrogen-and estrogen-induced feminization in fish. Aquatic Toxicology, 2007. 81(2): p. 219-231.

44. Roy, J.R., S. Chakraborty, and T.R. Chakraborty, Estrogen-like endocrine disrupting chemicals affecting puberty in humans--a review. Medical Science Monitor, 2009. 15(6): p. RA137-RA145.

45. Grün, F. and B. Blumberg, Perturbed nuclear receptor signaling by environmental obesogens as emerging factors in the obesity crisis. Reviews in Endocrine and Metabolic Disorders, 2007. 8(2): p. 161-171.

46. Orlando, E.F. and L.J. Guillette, Sexual dimorphic responses in wildlife exposed to endocrine disrupting chemicals. Environmental Research, 2007. 104(1): p. 163-173.

47. Arukwe, A., Steroidogenic acute regulatory (StAR) protein and cholesterol sidechain cleavage (P450scc)-regulated steroidogenesis as an organ-specific molecular and cellular target for endocrine disrupting chemicals in fish. Cell biology and toxicology, 2008. 24(6): p. 527-540. 
48. Dickerson, S.M. and A.C. Gore, Estrogenic environmental endocrine-disrupting chemical effects on reproductive neuroendocrine function and dysfunction across the life cycle. Reviews in Endocrine and Metabolic Disorders, 2007. 8(2): p. 143-159.

49. Dishaw, L.V., et al., Is the PentaBDE replacement, tris (1, 3-dichloro-2-propyl) phosphate (TDCPP), a developmental neurotoxicant? Studies in PC12 cells.

Toxicology and applied pharmacology, 2011. 256(3): p. 281-289.

50. Caballero-Gallardoa, K., J. Olivero-Verbela, and J.L. Freemanb, Supplementary Material Toxicogenomics to Evaluate Endocrine Disrupting Effects of Environmental Chemicals Using the Zebrafish Model. 